Vision in premature infants

\section{Refraction and keratometry in premature infants}

\section{X Repka}

\section{Normal development of corneal curvature is affected by preterm delivery}

A bnormalities of emmetropisation, resulting most often in myopia, have long been independently associated by numerous authors from nearly every corner of the globe with increasing prematurity, increasing severity of retinopathy of prematurity (ROP), and retinal ablative therapy. This topic has also been the subject of an editorial in the BJO in 1997, the substance of which remains true today. ${ }^{1}$ These refractive error abnormalities of prematurity and of ROP have been found to present early in infancy and persist into adulthood. It is not clear whether the mechanism for the development of myopia of prematurity is the same or different from the mechanism for the development of myopia of ROP. ${ }^{1}$ It appears that an alteration in the normal growth patterns of the eye is caused by the prematurity, the retinal ablative therapy, or probably both factors, leading to the refractive error. This disruption in growth results in structural abnormalities of parts of the eye which affect refractive development. These structures might include corneal curvature, axial length, lens thickness, and anterior chamber depth.

Most authors of studies involving myopia of prematurity have had only refractive error data available, which makes it impossible to directly determine which ocular structures have had their development altered before term. Furthermore, most studies have dealt with patients examined well beyond the expected due date, after which other environmental and disease factors could affect the observed outcome. ${ }^{23}$ There has been until recently few biometric data available for these premature patients to explain the refractive error findings, and essentially no such data during infancy. Donzis and coworkers reported a dramatic flattening in the corneal curvature of six premature patients with very steep corneas between 28-34 weeks gestation and term, which they associated with a change in refractive status. ${ }^{4}$ No comparison data to term infants were available, but these data can be used to support the idea that there is substantial, rapid corneal growth throughout this period. Cook and colleagues found, in premature infants without evidence of ROP (mean post-menstrual age at birth 29.4 weeks), or complex pattern of rapid growth between 30 and 50 weeks postmenstrual age. They noted a significant correlation between corneal curvature growth and refractive status resulting in less hypermetropia when compared to published data from term infants. ${ }^{5}$ Cook and her coworkers did not include comparison data for eyes of children delivered at term in their nursery or information concerning eyes with ROP.

\section{We do not know whether this change in corneal curvature mea- sured at term persists and can be held responsible for myopia of prematurity}

Snir and coworkers (p 900), in this issue of the $B J O$, have provided data that begin to fill two gaps in our understanding of the relation of prematurity to refractive error. They provide information about refraction, corneal curvature, and retinal appearance at 40 weeks post-menstrual age. In their design they studied two patient cohorts, one consisting of babies delivered and examined at term and a second group, delivered an average of 7.1 weeks before term (range 26-35 weeks), but who underwent their ocular evaluation at term. These authors determined refractive error with cycloplegic retinoscopy and corneal curvature with automated keratometry. Unfortunately, their design did not include other biometric measures such as axial length or anterior chamber depth. Funduscopy noted only mild ROP (stages 1 or 2 ) in $87.9 \%$ case, probably because very few infants less than $1000 \mathrm{~g}$ were included. Snir and colleagues found significantly less hypermetropia, about $1.1 \mathrm{D}$ on average, in the preterm infants compared to the term infants. They also found the corneas to be $1.5 \mathrm{D}$ steeper on average in the premature group. The similarity in magnitude of these two values when compared to term infants suggests that the alteration in the corneal curvature may be responsible for much or all of the observed change in refractive error state at 40 weeks post-menstrual age.

These data add substantial weight to the hypothesis that exposure to the extrauterine environment for a relatively short time before term significantly affects the growth pattern of the anterior segment. The rapidity of the change lends support to the hypothesis suggested by Fielder and colleagues nearly two decades ago. ${ }^{16}$ They postulated that the reduced temperature of the extrauterine eye leads to slowing of corneal growth, less flattening of the cornea, and thus less hypermetropia. The role of other factors such as light exposure and patterned visual stimulation for a short period before term on refractive development are not well studied in preterm infants. The similarity of the findings of Snir and colleagues for patients with mild ROP to those findings of Cook and her colleagues for patients without ROP suggests that the alterations in normal corneal curvature development before term may not be greatly affected by ROP.

The applicability of this report is limited by the lack of a wider birthweight distribution of premature infants, especially of infants less than $750 \mathrm{~g}$, and its similarly limited distribution of ROP severity, as almost every patient had stage 1 or 2 disease. These deficiencies make it impossible to generalise to smaller infants with greater ROP severity. Finally, the data from this study cannot confirm that corneal curvature is alone responsible for the epidemic of myopia seen among extreme low birthweight infants and those with severe ROP as, here again, insufficient numbers of such patients were included in this study and other biometric factors were not studied. Anterior chamber depth seems to be an important factor, ${ }^{57}$ and other factors such as lens thickness and axial elongation remain to be clarified by future investigation. A further limitation is the lack of biometric data on this cohort of patients later in the first year of life. Thus, we do not know whether this change in corneal curvature measured at term persists and can be held responsible for myopia of prematurity. ${ }^{2}$

This study has confirmed that the normal development of corneal curvature is affected by preterm delivery producing less hypermetropia in the setting of mild ROP. The direct cause for this change is unknown. As for the development of myopia of prematurity and myopia of ROP, it is likely that additional modifying factors following 
delivery, both in the nursery and in the home environment, has a large role in the refractive error outcome measured at 3 months of age and beyond.

Br J Ophthalmol 2004;88:853-854.

doi: 10.1136/bjo.2003.041186

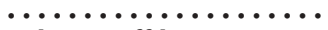

Authors' affiliations

M X Repka, Krieger Children's Eye Center, 600 N Wolfe Street, Baltimore, MD 21287,

USA; mrepka@jhmi.edu

\section{REFERENCES}

1 Fielder AR, Quinn GE. Myopia of prematurity: nature, nurture, or disease? $\mathrm{Br} J$ Ophthalmol 1997;81:2-3.

2 Quinn GE, Dobson V, Siatkowski RM, et al. Does cryotherapy affect refractive error? Results from treated versus control eyes in the cryotherapy for retinopathy of prematurity trial. Ophthalmology 2001:108:343-7.

3 Larsson EK, Rydberg AC, Holmstrom GE.

A population-based study of the refractive outcome in 10-year-old preterm and full-term children. Arch Ophthalmol 2003; 121:1430-6.
4 Donzis PB, Insler MS, Gordon RA. Corneal curvatures in premature infants. Am J Ophthalmol 1985;99:213-5.

5 Cook A, White S, Batterbury M, et al. Ocular growth and refractive error development in premature infants without retinopathy of prematurity. Invest Ophthalmol Vis Sci 2003:44:953-60.

6 Fielder AR, Levene MI, Russell-Eggitt IM, et al. Temperature-a factor in ocular development? Dev Med Child Neurol 1986;28:279-84.

7 Hittner HM, Rhodes LM, McPherson A. Anterior segment abnormalities in cicatricial retinopathy of prematurity. Ophthalmology 1979;86:803-16.
Glaucoma

\section{Treatment of congenital cataract}

\section{S R Lambert}

\section{It may all come down to timing}

$\mathrm{T}$ he visual prognosis for children with congenital cataracts has improved dramatically since it was first recognised that cataract surgery during infancy is critical for a good visual outcome ${ }^{2}$; however, the optimal time to perform cataract surgery in a child with a congenital cataract is still in question. Anecdotal reports have noted excellent visual outcomes in neonates undergoing cataract surgery during the first week of life ${ }^{13}$; however, a subsequent analysis of 45 children with dense unilateral cataracts, who underwent cataract surgery, found that the visual outcome was the same regardless of when the surgery was performed during the first 6 weeks of life. ${ }^{4}$ While the same analysis has not been performed on children with bilateral congenital cataracts, it has been proposed that the critical period for treating children with bilateral congenital cataracts may extend to 8 weeks of life. ${ }^{256}$ Paediatric ophthalmologists are now trying to determine the optimal time to perform cataract surgery to reduce the high incidence of complications that have been reported following cataract surgery during infancy.

One of the most serious complications that occurs following paediatric cataract surgery is glaucoma. It is usually open angle and has been reported to develop in up to one third of children after a lensectomy and vitrectomy. ${ }^{7-11}$ Known risk factors for aphakic glaucoma include microcornea, persistent fetal vasculature, and cataract surgery during infancy. Parks et al ${ }^{8}$ reported that 54\% of the children in their series who developed aphakic glaucoma underwent cataract surgery when they were younger than 2 months of age. Rabiah $^{11}$ reported that $37 \%$ of children undergoing cataract surgery when 9 months of age or younger developed glaucoma compared to only $6 \%$ of children undergoing surgery thereafter. Vishwanath et al reports in this issue of the BJO (p 905) that $50 \%$ of children undergoing bilateral lensectomies during the first month of life developed glaucoma in one or both eyes after a 5 year follow up compared to only 15\% of children undergoing cataract surgery thereafter. Lundvall and Kugelberg ${ }^{5}$ also reported that $80 \%$ of the children in their series of children with bilateral congenital cataracts who developed glaucoma underwent cataract surgery during the first 4 weeks of life. Finally, Watts and coworkers ${ }^{12}$ reported that aphakic glaucoma was more prevalent in children undergoing surgery when 14-34 days of age. While it is possible that eyes with congenital cataracts requiring early surgery are more prone to develop glaucoma than eyes that acquire cataracts later in childhood, it is also likely that early surgery increases the risk of these eyes developing glaucoma. For this reason, Vishwanath and coworkers have proposed that cataract surgery should be deferred until after the first 4 weeks of life.

It would appear that the optimal time to remove a dense congenital cataract in an infant and to initiate optical treatment is when the child is 4-6 weeks of age

The pathophysiology of aphakic open angle glaucoma is poorly understood. Its aetiology has been attributed to damage to the trabecular meshwork by inflammation, the loss of mechanical support of the trabecular meshwork, or a toxic substance gaining access to the trabecular meshwork from the vitreous humour. ${ }^{13}$ It has been reported to occur after both limbal and a pars plicata based lensectomies, suggesting that the surgical approach is not the critical factor in determining its occurrence. ${ }^{14}$

Children are more commonly being optically corrected with intraocular lenses following cataract surgery. ${ }^{15}$ Asrani and coworkers ${ }^{13}$ have reported a decreased incidence of open angle glaucoma in older children undergoing primary IOL implantation following cataract surgery. Recent studies, however, have noted a high incidence of open angle glaucoma in children undergoing IOL implantation during the first 4 weeks of life. ${ }^{16-18}$

A final factor to consider is the safety of general anaesthesia during infancy. The immaturity of an infant's cardiovascular, pulmonary, and gastrointestinal systems, as well as their liver, kidneys, and thermoregulation puts them at greater risk from general anaesthesia. ${ }^{19}$ The most life threatening risk to an infant is postoperative apnoea. The risk of postoperative apnoea has been shown to be higher in preterm infants less than 44 post-conception weeks of age..$^{20}$ For this reason, it has been recommended that all non-essential surgery for preterm infants be delayed until they are older than 44 post-conception weeks of age. ${ }^{21}$ While an increased risk of postoperative apnoea in a full term infant following general anaesthesia has not been established, waiting until an infant is older to perform cataract surgery allows all of the different systems in an infant to mature to some degree and should be safer.

From the available data, it would appear that the optimal time to remove a dense congenital cataract in an infant and to initiate optical treatment is when the child is 4-6 weeks of age. To remove the cataract before 4 weeks of age appears to increase the risk of the eye developing aphakic glaucoma, whereas 
waiting beyond 6 weeks of age compromises the visual outcome.

Br J Ophthalmol 2004;88:854-855.

doi: 10.1136/bjo.2004.045401

Correspondence to: S R Lambert, Emory Eye Center, 1365-B, Clifton Road, NE, Atlanta, GA 30322, USA; slamber@emory.edu

Accepted for publication 26 February 2004

Supported in part by NIH Core Grant EY06360 and Research to Prevent Blindness, Inc.

\section{REFERENCES}

1 Beller R, Hoyt CS. Good visual function after neonatal surgery for congenital monocular cataracts. Am J Ophthalmol 1981;91:559-65.

2 Gelbart SS, Hoyt CS, Jastrebski G, et al. Longterm visual results in bilateral congenital cataracts. Am J Ophthalmol 1982;93:615-21.

3 Gregg FM, Parks MM. Stereopsis after congenital monocular cataract extraction. Am J Ophthalmol 1992:114:314-17.

4 Birch EE, Stager DR. The critical period for surgical treatment of dense congenital unilateral cataract. Invest Ophthalmol Vis Sci 1996;37:1532-8.
5 Lundvall A, Kugelberg U. Outcome after treatment of congenital bilateral cataract. Acta Ophthalmol Scand 2002;80:593-7.

6 Magnusson G, Abrahamsson M, Siöstrand J. Changes in visual acuity from 4 to 12 years of age in children operated for bilateral congenital cataracts. Br J Ophthalmol 2002;86:1385-9.

7 Simon JW, Mehta N, Simmons S, et al. Glaucoma after pediatric lensectomy/vitrectomy. Ophthalmology 1991;98:670-4.

8 Parks MM, Johnson DA, Reed GW. Long-term visual results and complications in children with aphakia. A function of cataract type. Ophthalmology 1993;100:826-41.

9 Egbert JE, Wright MM, Dahlhauser KF, et al. A prospective study of ocular hypertension and glaucoma after pediatric cataract surgery. Ophthalmology, 102:1098-101.

10 Johnson CP, Keech RV. Prevalence of glaucoma after surgery for PHPV and infantile cataracts. J Pediatr Ophthalmol Strabismus 1996;33:14-17.

11 Rabiah PK. Frequency and predictors of glaucoma after pediatric cataract surgery. Am J Ophthalmol 2004;137:30-7.

12 Watts $\mathbf{P}$, Abdolell M, Levin AV. Complications in infants undergoing surgery for congenital cataract in the first 12 weeks of life: Is early surgery better? J AAPOS 2003;7:81-5.

13 Asrani S, Freedman S, Hasselblad V, et al. Does primary intraocular lens implantation prevent

\section{Trabeculectomy and antimetabolites}

\section{$S$ Fraser}

\section{Perfecting a technique that works takes practice, but it is getting increasingly hard to get this practice}

W hen von Graefe first introduced iridectomy as a treatment for glaucoma, some practitioners noted that the patients who usually had persistent reductions in intraocular pressure (IOP) were those who developed an inadvertent filtering bleb at the site of the surgery. This led De Wecker, ${ }^{1}$ in 1882, to suggest that the IOP could be reduced by purposely creating a fistula between the anterior chamber and the subconjunctival tissues. He was the first to use the term "filtering" to describe this egress of fluid from the eye. ${ }^{2}$

Full thickness filtering procedures were used throughout the early 20th century but were bedevilled by a high rate of postoperative complications such as hypotony, flat anterior chambers, and suprachoroidal haemorrhage. For this reason, in the 1960s, a guarded filtration technique was developed and (incorrectly) named trabeculectomy. ${ }^{3}{ }^{4}$ It is a tribute to this operation that it has, with certain modifications, persisted to the present day.

However, just as full thickness filtering procedures were compromised by excessive drainage, the enemy with guarded drainage procedures has been excessive scarring with subsequent reduction or abolition of aqueous flow from the eye into the subconjunctival space. It is now well recognised that in some situations this risk of failure secondary to scarring is greater. These include those aged under 40, those of African-Caribbean descent, and those with previous ocular inflammation including that induced by surgery and topical medications.

This scarring response has long been known to compromise filtration surgery-many of the advocates of de Wecker's "filtering cicatrices" were disappointed that the lowering of the IOP rarely lasted more than 1 year. ${ }^{2}$ But it is only relatively recently that we have been able to modify this healing response with the use of the antimetabolites 5-fluorouracil (5-FU) and mitomycin C (MMC)..$^{56}$

The paper by Siriwardena et al, in this issue of BJO (p 873), indicates how important a tool these antimetabolites have become, with $82 \%$ of UK consultants who responded to their survey stating that they used them. What is perhaps more surprising is that only $9 \%$ used antimetabolites in more than half "aphakic" glaucoma in children? J AAPOS 1999;3:33-9.

14 Muñoz M, Parrish RK, Murray TG. Open-angle glaucoma after pars plicata lensectomy and vitrectomy for congenital cataracts. Am J Ophthalmol 1995; 119:103-4.

15 Lambert SR, Lynn M, Drews-Botsch C, et al. Intraocular lens implantation during infancy: Perceptions of parents and the American Association for Pediatric Ophthalmology and Strabismus members. J AAPOS 2003;7:400-5.

16 Lambert SR, Buckley EG, Plager DA, et al. Unilateral intraocular lens implantation during the first six months of life. J AAPOS the first six mon
1999:3:344-9.

17 Wilson ME, Peterseim MW, Englert JA, et al. Pseudophakia and polypseudophakia in the first year of life. J AAPOS 2001:5:238-45.

18 Plager DA, Yang S, Neely D, et al. Complications in the first year following cataract surgery with and without $\mathrm{IOL}$ in infants and older children. J AAPOS 2002;6:9-14.

19 Miller RD. Developmental physiology of the infant. In: Anesthesia. 5th ed. Edinburgh: Churchill Livingstone, 2000:2089-92.

20 Wellborn LG, Ramirez N, Oh TH, et al. Postanesthetic apnea and periodic breathing in infants. Anesthesiology 1986;65:658.

21 Gregory GA, Steward DJ. Life-threatening perioperative apnea in the ex" $x^{\prime \prime}$ premie" $^{\prime \prime}$ (editorial). Anesthesiology 1983;59:495.

their cases. Similarly only $2 \%$ (that is, less than 20) of UK consultants used MMC in more than half their cases. Although this survey reflects practice in 1999 it appears that this figure is substantially lower than those for the United States and Japan.

Trabeculectomy will become a subspecialist procedure performed by those who have completed a recognised period of training in glaucoma and who perform a minimum number of trabeculectomies per annum

This discrepancy may well reflect differences in case mix between different countries-especially in the United States with a much higher black population. It may also reflect a more conservative approach in the United Kingdom. The complications of antimetabolite use are well known, including cataract, prolonged hypotony, epitheliopathy, blebitis, and endophthalmitis. ${ }^{78}$ Trabeculectomy is usually performed on eyes with normal visual acuity and vision threatening results are devastating to even the most fully informed patient.

As all ophthalmologists know, filtration surgery represents a delicate balance between maintaining the integrity of the eye and prevention of excess scarring. Although, like all surgery there is an element we cannot control, the key to successful surgery is to perfect a technique that allows as much control over the eye as possible. This involves preoperative assessments of risks (for example, reducing conjunctival inflammation 
with steroids), a meticulous intraoperative technique (for example, use of antimetabolites, releasable sutures), and timely and appropriate postoperative interventions (for example, subconjunctival 5-FU, bleb needling). It is consideration of these factors that allows us to sit within the therapeutic window that produces a sighted eye with an appropriate level of intraocular pressure.

Perfecting a technique that works takes practice-surgeons who do more of a particular type of operation tend to get better results. ${ }^{9}$ It is, however, getting increasingly hard to get this practice. The number of trabeculectomies performed in the United Kingdom has declined over the past 5 years. ${ }^{1011}$ There may be a number of reasons for this, including a wider range of medical treatments, earlier diagnosis, or perhaps a reduction in the number of redo trabeculectomies because of the use of antimetabolites. Because of this, ophthalmology trainees are now less likely to be exposed to filtration surgery and at the end of their higher specialist training in ophthalmology (www.rcophth.ac.uk/ education/curriculum hst_ophth.html) are unlikely to be fully competent in the procedure.

It is inevitable that, with fewer trabeculectomies being performed, patients are on topical treatments for longer. Because of the effects of topical medications on the conjunctiva it is likely that, in those patients coming to filtering surgery, antimetabolites will more often be used, making the surgery less forgiving. With the added pressure of increased public scrutiny of doctors and a generally more aware patient group it would appear inevitable, whether we like it or not, that trabeculectomy will become a subspecialist procedure performed by those who have completed a recognised period of training in glaucoma and who perform a minimum number of trabeculectomies per annum.

Br J Ophthalmol 2004;88:855-856.

doi: $10.1136 /$ bjo.2004.040055

Correspondence to: Mr Scott Fraser,

Sunderland Eye Infirmary, Queen Allexandra Road, Sunderland SR2 9HP, UK; sfraser100@ totalise.co.uk

\section{REFERENCES}

1 De Wecker L. La cicatrice a filtration. Ann Oculist 1882:133-43.

2 Albert DM, Edwards DD. The history of ophthalmology-glaucoma. 1 st ed. Oxford: Blackwell Science, 1996.

3 Sugar HS. Experimental trabeculectomy in glaucoma. Am J Ophthalmol 1961;51:623-7.

4 Cairns JE. Trabeculectomy. Preliminary report of a new method. Am J Ophthalmol 1968:66:673-8.

5 Hever DK, Parrish RKI, Gressel MG. 5Fluorouracil and glaucoma filtration surgery. II. A pilot study. Ophthalmology 1984;91:384-94.

6 Chen CW. Enhanced intraocular pressure controlling effectiveness of trabeculectomy by local application of mitomycin-C. Trans AsiaPacific Acad Ophthalmol 1983;9:172.

7 Wormald R, Wilkins MR, Bunce C. Post-operative 5-fluorouracil for glaucoma surgery (Cochrane review). In: The Cochrane Library, Issue 4, 2003. Chichester, UK: John Wiley \& Sons.

8 Bindlish R, Condon GP, Schlosser JD, et al. Efficacy and safety of mitomycin-C in primary trabeculectomy. Ophthalmology 2002;109:1336-41.

9 Soljak M. Volume of procedures and outcome of treatment. BMJ 2002;325:787-8.

10 Whittaker KW, Gillow JT, Cunliffe IA. Is the role of trabeculectomy in glaucoma management changing? Eye $2001 ; 15: 449-52$.

11 Bateman DN, Clark R, Azuara-Blanco A, et al. The impact of new drugs on management of glaucoma in Scotland: observational study. BM 2001;323:1401-2.

New RPGR gene mutation produces an extended X linked RP syndrome

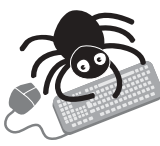

Please visit the British Journal of

Ophthalmology website [www. bjophthalmol. com] for a link to the full text to the full text
of this article.
$D$ r esearchers have warned against equating animal and human models of X linked retinitis pigmentosa (XLRP) after finding a new mutation in the retinitis pigmentosa GTPase regulator (RPGR) gene associated with a unique, extended XLRP syndrome in one family. The syndrome encompasses recurrent middle ear and upper respiratory tract infections (URTIs), hearing loss, and impaired sight. Compromised ciliary function may be the cause.
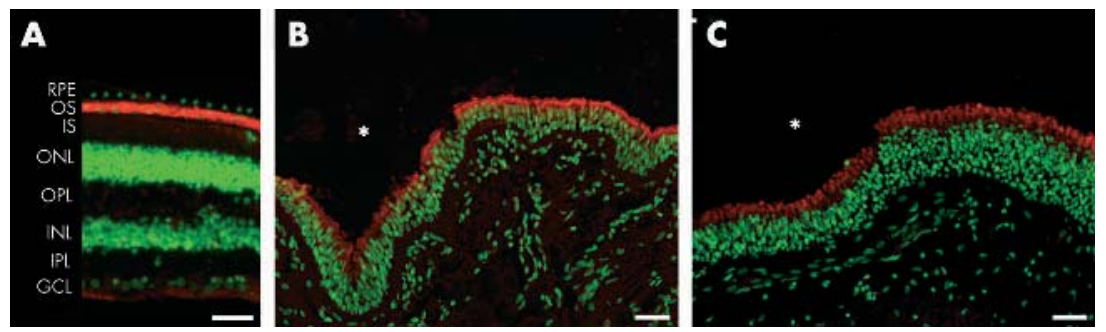

Evidence of expression of native GTPase regulator by red staining on immunofluorescence with specific antibody in sections of normal human tissue. Staining confined to the photoreceptor outer segment area (OS) in retina (A); in epithelial cells lining the lumen in bronchi (B); and sinuses (C). Asterisks indicate lumen in (B) and (C). Bar $=100 \mu \mathrm{m}$.

The researchers found a G576C substitution in the RPGR gene, causing a glycine to arginine shift in the GTPase regulator in the proband-a 10 year old white boy with hearing loss referred for confirmation of suspected RP or Usher syndrome, present in a relative four generations previously. The mutation occurred in his younger brother, who had recurrent URTIs and ear infections and was developing hearing loss and sight problems; their maternal grandmother, who had late RP and 15 years' hearing loss after decades of recurrent middle ear infections; and their mother, who had no symptoms. It was absent in the boys' father and sister, who were symptom free, and in over 200 normal human chromosomes.

Native GTPase regulator was expressed in human retinal, bronchial, and sinus epithelium and in human and monkey cochlea epithelium, consistent with the range of phenotypic symptoms in subjects with the mutation.

The findings were based on full ophthalmological examinations; hearing tests; clinical data on infections from patient records; and molecular genetic analysis in the six key family members.

Hearing loss and recurrent middle ear and upper respiratory tract infections are rare in XLRP.

A Journal of Medical Genetics 2003;40:118 (http://www.jmedgenet.com/cgi/content/full/40/11/118) 